IPMU09-0066

\title{
Dark matter as integration constant in Hořava-Lifshitz gravity
}

\author{
Shinji Mukohyama \\ Institute for the Physics and Mathematics of the Universe (IPMU) \\ The University of Tokyo \\ 5-1-5 Kashiwanoha, Kashiwa, Chiba 277-8582, Japan
}

\begin{abstract}
In the non-relativistic theory of gravitation recently proposed by Hořava, the Hamiltonian constraint is not a local equation satisfied at each spatial point but an equation integrated over a whole space. The global Hamiltonian constraint is less restrictive than its local version, and allows a richer set of solutions than in general relativity. We show that a component which behaves like pressureless dust emerges as an "integration constant" of dynamical equations and momentum constraint equations. Consequently, classical solutions to the infrared limit of Hořava-Lifshitz gravity can mimic general relativity plus cold dark matter.
\end{abstract}




\section{Introduction}

Dark energy and dark matter are two major mysteries in modern cosmology. Assuming that general relativity is correct at long distances up to cosmological scales, precision observational data indicates that more than $90 \%$ of our universe consists of dark energy and dark matter. Although some gravitational properties of the dark components are known, they are not optically observed and, thus, we do not know what they really are. This situation makes us suspect that modifying gravity in the infrared (IR) might address the mysteries of dark energy and/or dark matter.

Recently a power-counting renormalizable 1 theory of gravitation was proposed by Hořava [1, 2]. One of the most important aspects of the theory is that in the ultraviolet (UV) it is fundamentally non-relativistic and exhibits the Lifshitz scale invariance

$$
t \rightarrow b^{z} t, \quad \vec{x} \rightarrow b \vec{x}
$$

with dynamical critical exponent $z=3$. Hořava's theory is considered as a potential candidate for the theory of quantum gravity and is often called Hořava-Lifshitz gravity. Various aspects of this theory have been investigated [3]- [40].

Hořava-Lifshitz gravity has not yet been intended to be a unified theory. Clearly, further developments or/and embedding into a "bigger" theory is needed. For example, since the "limit of speed" is an emergent quantity in the IR, different species including those in the standard model of particle physics must be related to each other in the framework of Hořava's theory so that the "limits of speed" for different species in the IR agree with the "velocity of light" 2. This obviously indicates that embedding of this theory into a unified theory (or other way around) is necessary for the theory to be a part of the real world.

Still, it is interesting to investigate universal properties of the theory 3 and its cosmological implications, in parallel with those fundamental issues. For example, the $z=3$ Lifshitz scaling not only is the origin of the power-counting renormalizability but also leads to a number of interesting cosmological consequences, such as generation of scale-invariant cosmological perturbations from a non-inflationary epoch of the early universe [7] and a particular scaling of radiation energy density $\left(\propto a^{-6}\right)[19$ ].

The purpose of the present paper is to point out that Hořava-Lifshitz gravity can

\footnotetext{
${ }^{1}$ Note, however, that renormalizability has not yet been established in a rigorous manner beyond the level of power-counting.

${ }^{2}$ See e.g. refs. [41, 42, 43] for tight experimental limits on Lorentz violation.

${ }^{3}$ In this respect, the so called detailed balance condition is neither essential nor universal for Hořava-Lifshitz gravity as already stated in [1].
} 
mimic general relativity plus cold dark matter.

\section{Basic idea}

Before explaining why the IR limit of Hořava-Lifshitz gravity can behave like general relativity plus cold dark matter, let us remind ourselves about the structure of Einstein's general relativity since the existence of dark matter was suspected by assuming general relativity. General relativity fully respects 4-dimensional spacetime diffeomorphism invariance as the fundamental symmetry of the theory. As a result, it has four constraint equations: one called Hamiltonian constraint and three called momentum constraint. These constraints must be satisfied at each spatial point at each time. However, since the constraint equations are preserved under time evolution by dynamical equations, i.e. other components of the Einstein equation, it is also possible to impose the constraint equations only on an initial hypersurface and to solve dynamical equations afterwards. In this case, constraint equations are automatically satisfied at late time.

As an illustration, let us consider a flat Friedmann-Robertson-Walker (FRW) spacetime driven by components with equations of state $P_{i}=P_{i}\left(\rho_{i}\right)$, where $\rho_{i}$ and $P_{i}$ are energy density and pressure of the $i$-th component. Because of the spatial homogeneity, the momentum constraint is trivial. On the other hand, the Hamiltonian constraint gives the famous Friedmann equation:

$$
3 \frac{\dot{a}^{2}}{a^{2}}=8 \pi G_{N} \sum_{i=1}^{n} \rho_{i},
$$

where $a$ is the scale factor of the universe, a dot represents time derivative and $n$ is the number of components. The conservation of stress energy tensor states that

$$
\dot{\rho}_{i}+3 \frac{\dot{a}}{a}\left(\rho_{i}+P_{i}\right)=0
$$

These $n+1$ equations are sufficient to predict future evolution of the universe, provided that the initial value of $a$ and $\rho_{i}$ are specified. The remaining non-trivial component of the Einstein equation gives the dynamical equation

$$
-2 \frac{\ddot{a}}{a}-\frac{\dot{a}^{2}}{a^{2}}=8 \pi G_{N} \sum_{i=1}^{n} P_{i},
$$

but this follows from the previous $n+1$ equations. Therefore, it suffices to solve the Friedmann equation (2.1) coupled with the conservation equation (2.2). However, it is also consistent to solve the dynamical equation (2.3) coupled with the conservation 
equation (2.2), provided that the Friedmann equation (2.1) is imposed at an initial time. In other words, the Friedmann equation can be considered as an first integral of the dynamical equation with a special choice of an integration constant.

Now, let us suppose that there is a theory without Hamiltonian constraint. Let us, however, suppose that in the FRW spacetime, we still have the conservation equation (2.2) and the dynamical equation (2.3) . This is perfectly fine as we have $n+1$ independent differential equations for $n+1$ variables, $a(t)$ and $\rho_{i}(t)(i=1, \cdots, n)$. Actually, we obtain

$$
3 \frac{\dot{a}^{2}}{a^{2}}=8 \pi G_{N}\left(\sum_{i=1}^{n} \rho_{i}+\frac{C}{a^{3}}\right)
$$

as a first integral of the dynamical equation, where $C$ is an integration constant, and this is almost the same as the Friedmann equation (2.1). The only difference is the term $C / a^{3}$. What is interesting is that this is exactly of the form of dark matter. In general relativity, dark matter $\left(\propto a^{-3}\right)$ is included as one of $\rho_{i}$ 's and, thus, must be derived from an action principle since $\rho_{i}$ is a component of a stress-energy tensor. In general relativity this is the origin of the mystery: we need to explain what dark matter is made of by specifying its action. On the other hand, in this hypothetical theory without Hamiltonian constraint, the term proportional to $a^{-3}$ emerges as an integration constant and, thus, we do not need an action for it.

Intriguingly enough, as we shall briefly explain in the next paragraph, in HořavaLifshitz gravity there is no Hamiltonian constraint as a local equation at each spatial point. Instead, the Hamiltonian constraint equation in Hořava-Lifshitz gravity is an equation integrated over a whole space. In homogeneous spacetime such as the FRW spacetime, the global Hamiltonian constraint is as good as local one since all spatial points are equivalent. However, in inhomogeneous spacetimes there can be drastic differences. If the whole universe is much larger than the present Hubble volume then it is possible that the universe far beyond the present Hubble horizon is different from our patch of the universe inside the horizon. In this case, the global Hamiltonian constraint does not restrict the universe inside the horizon. Even if we approximate our patch of the universe inside the present horizon by the FRW spacetime, the whole universe can include inhomogeneities of super-horizon scales and, thus, the global Hamiltonian constraint does not restrict the FRW spacetime which just approximates the behavior inside the horizon. Therefore, as in the hypothetical theory considered in the previous paragraph, the absence of local Hamiltonian constraint in HorravaLifshitz gravity results in an extra term $\propto a^{-3}$ in the "Friedmann equation" or, to be precise, the first integral of the dynamical equation. As before, this term can mimic dark matter but we do not need an action for it. 
Absence of local Hamiltonian constraint in Hořava-Lifshitz gravity originates from the projectability of the lapse function. The basic quantities in Hořava-Lifshitz gravity are the 3-dimensional spatial metric $g_{i j}$, the shift vector $N^{i}$ and the lapse function $N$. In terms of these quantities the 4-dimensional spacetime metric is written in the ADM form:

$$
d s^{2}=-N^{2} d t^{2}+g_{i j}\left(d x^{i}+N^{i} d t\right)\left(d x^{j}+N^{j} d t\right) .
$$

The former two, $g_{i j}$ and $N^{i}$, can depend on both spatial coordinates $x^{k}$ and the time variable $t$. On the other hand, the projectability condition states that the lapse function $N$ should depend only on $t$ and be independent of spatial coordinates. The projectability of the lapse function stems from the fundamental symmetry of the theory, i.e. invariance under the foliation-preserving diffeomorphism:

$$
x^{i} \rightarrow \tilde{x}^{i}\left(x^{j}, t\right), \quad t \rightarrow \tilde{t}(t)
$$

and therefore must be respected. Essentially, the lapse function $N$ represents a gauge degree of freedom associated with the space-independent time reparametrization. Thus, it is very natural to restrict $N$ to be independent of spatial coordinates. This is the projectability condition. Of course, a space-independent $N$ cannot be transformed to a space-dependent function by foliation-preserving diffeomorphism. This point was already made clear by Hořava [1, 4.

\section{IR limit of Hořava-Lifshitz gravity}

In the IR limit the action of Hořava-Lifshitz gravity is reduced to

$$
I_{H L}=\frac{1}{16 \pi G_{N}} \int d t d^{3} x \sqrt{g} N\left[K_{i j} K^{i j}-\lambda K^{2}+R-2 \Lambda\right]
$$

where $K_{i j}=\left(\dot{g}_{i j}-D_{i} N_{j}-D_{j} N_{i}\right) /(2 N)$ is the extrinsic curvature of the constant time hypersurface, $K=K_{i}^{i}$, and $D$ is the 3 -dimensional covariant derivative compatible with $g_{i j}$. This looks identical to the Einstein-Hilbert action in the ADM form if and only if $\lambda=1$. Hence, hereafter, we assume that the renormalization group (RG) flow brings $\lambda$ to 1 in the IR or that $\lambda$ stays at 1 from higher energy scales all the way down to the IR under the RG flow. The RG flow of Hořava-Lifshitz gravity has not been investigated in details and, thus, must be addressed in the future. In this paper, we simply assume that $\lambda=1$ is an IR fixed point of the RG flow.

\footnotetext{
${ }^{4}$ In the last paragraph of subsection 2.1 of [1], it says that, except for the case with extra symmetry such as the Weyl symmetry $(\lambda=1 / 3)$, fluctuations of the lapse function must be space-independent.
} 
Even with $\lambda=1$, however, there is an important difference between the IR limit of Hořava-Lifshitz gravity and general relativity. In Hořava-Lifshitz gravity the projectability condition requires that the lapse function $N$ should depend only on $t$. Because of this restriction, the Hamiltonian constraint, i.e. the equation derived from functional derivative of the total action with respect to the lapse function, is not a local equation but an equation integrated over a constant time hypersurface:

$$
\int d^{3} x \sqrt{g}\left(G_{\mu \nu}^{(4)}+\Lambda g_{\mu \nu}^{(4)}-8 \pi G_{N} T_{\mu \nu}\right) n^{\mu} n^{\nu}=0 .
$$

Here, $g_{\mu \nu}^{(4)}$ is the 4-dimensional metric shown in (2.5), $G_{\mu \nu}^{(4)}$ is the corresponding 4dimensional Einstein tensor, $T_{\mu \nu}$ is the stress energy tensor, and $n^{\mu}$ is the unit normal to the constant time hypersurface given by

$$
n_{\mu} d x^{\mu}=-N d t, \quad n^{\mu} \partial_{\mu}=\frac{1}{N}\left(\partial_{t}-N^{i} \partial_{i}\right)
$$

On the other hand, the momentum constraint and the dynamical equations are local equations as in general relativity:

$$
\left(G_{i \mu}^{(4)}+\Lambda g_{i \mu}^{(4)}-8 \pi G_{N} T_{i \mu}\right) n^{\mu}=0
$$

and

$$
G_{i j}^{(4)}+\Lambda g_{i j}^{(4)}-8 \pi G_{N} T_{i j}=0
$$

The Hamiltonian constraint (3.2) and momentum constraint (3.4) are preserved by the dynamical equations (3.5). Thus, it suffices to solve the dynamical equations, provided that the initial condition satisfies the constraint equations. Note that the global Hamiltonian constraint (3.2) is less restrictive than its local version, and allows a richer set of solutions than in general relativity.

\section{Dark matter as "integration constant"}

Let us define deviation from general relativity $T_{\mu \nu}^{H L}$ by

$$
T_{\mu \nu}^{H L} \equiv \frac{1}{8 \pi G_{N}}\left(G_{\mu \nu}^{(4)}+\Lambda g_{\mu \nu}^{(4)}\right)-T_{\mu \nu}
$$

or

$$
G_{\mu \nu}^{(4)}+\Lambda g_{\mu \nu}^{(4)}=8 \pi G_{N}\left(T_{\mu \nu}+T_{\mu \nu}^{H L}\right)
$$

This looks like Einstein equation with the dark sector $T_{\mu \nu}^{H L}$. In the IR, not only the gravitational sector but also the (real) matter sector should respect the full 4dimensional diffeomorphism invariance. Thus, the conservation of energy momentum 
tensor for real matter $\nabla^{\mu} T_{\mu \nu}=0$ should hold in the IR, where $\nabla$ is the 4-dimensional covariant derivative compatible with $g_{\mu \nu}^{(4)}$. The Bianchi identity then implies the conservation $\nabla^{\mu} T_{\mu \nu}^{H L}=0$ of the dark sector. If the 4-dimensional diffeomorphism invariance is slightly broken in the (real) matter sector then $\nabla^{\mu} T_{\mu \nu}^{H L}=-\nabla^{\mu} T_{\mu \nu} \neq 0$. Note that invariance of the (real) matter action under 3-dimensional spatial diffeomorphism implies that $\nabla^{\mu} T_{\mu i}=0$ and thus $\nabla^{\mu} T_{\mu \nu} \propto n_{\nu}$.

The field equations in the IR limit of Horava-Lifshitz gravity is now written in terms of $T_{\mu \nu}^{H L}$. The Hamiltonian constraint (3.2) is

$$
\int d^{3} x \sqrt{g} T_{\mu \nu}^{H L} n^{\mu} n^{\nu}=0 .
$$

The momentum constraint (3.4) and dynamical equations (3.5) are

$$
T_{i \mu}^{H L} n^{\mu}=0
$$

and

$$
T_{i j}^{H L}=0 .
$$

As a general solution to the momentum constraint and dynamical equations, we obtain

$$
T_{\mu \nu}^{H L}=\rho^{H L} n_{\mu} n_{\nu}
$$

where $\rho^{H L}$ is a scalar function of spacetime coordinates $\left(t, x^{i}\right)$. This is equivalent to the stress energy tensor of a pressureless dust with energy density $\rho^{H L}$ and the unit tangent $n^{\mu}$ to its flow. Note that $n^{\mu}$ is tangent to a congruence of geodesics:

$$
n^{\mu} \nabla_{\mu} n_{\nu}=n^{\mu} \nabla_{\nu} n_{\mu}=\frac{1}{2} \partial\left(n^{\mu} n_{\mu}\right)=0 .
$$

Here, for the first equality, we have used the expression (3.3) and the fact that the lapse function $N$ depends only on $t$. Finally, the Hamiltonian constraint is

$$
\int d^{3} x \sqrt{g} \rho^{H L}=0 .
$$

This states that the total energy of the dust-like component in the dark sector should vanish. Of course $\rho^{H L}$ can be positive everywhere in our patch of the universe. As already explained in Sec. 2, this applies even when we approximate our universe by a FRW spacetime since overall homogeneity inside the current Hubble volume does not exclude super-horizon inhomogeneities.

As stated at the end of the previous section, the dynamical equations preserve the constraint equations. Thus, it suffices to solve the "modified Einstein equation"

$$
G_{\mu \nu}^{(4)}+\Lambda g_{\mu \nu}^{(4)}=8 \pi G_{N}\left(T_{\mu \nu}+\rho^{H L} n_{\mu} n_{\nu}\right)
$$


coupled with field equations of real matter fields, provided that the initial condition of the dark sector satisfies the global Hamiltonian constraint (4.8). Note that $\rho^{H L}$ does not have to vanish everywhere. It can be positive somewhere in the universe and negative elsewhere, as far as it sums up to zero. For example, $\rho^{H L}$ can be positive everywhere in our patch of the universe inside the present Hubble horizon. Note also that the additional term $\rho^{H L} n_{\mu} n_{\nu}$ is just an "integration constant" and does not represent a real dust. In other words, this additional term acts as cold dark matter but does not require an action.

The Bianchi identity applied to the "modified Einstein equation" (4.9) leads to

$$
\left(\partial_{\perp} \rho^{H L}+K \rho^{H L}\right) n_{\nu}=-\nabla^{\mu} T_{\mu \nu}
$$

where $\partial_{\perp}=n^{\mu} \partial_{\mu}$ and $\nabla$ is the 4-dimensional covariant derivative compatible with $g_{\mu \nu}^{(4)}$. This is consistent with the fact that the 3 -dimensional spatial diffeomorphism invariance of the (real) matter action implies $\nabla^{\mu} T_{\mu \nu} \propto n_{\nu}$. Therefore, we obtain

$$
\partial_{\perp} \rho^{H L}+K \rho^{H L}=n^{\nu} \nabla^{\mu} T_{\mu \nu} .
$$

The right hand side acts as a source term for the "dark matter" energy density $\rho^{H L}$, and is non-vanishing if the (real) matter sector breaks a part of 4-dimensional diffeomorphism invariance.

If the gravity sector also breaks the 4-dimensional diffeomorphism, i.e. if $\lambda$ slightly deviates from 1 or/and higher spatial curvature terms become non-negligible, then the left hand side of the "modified Einstein equation" (4.9) gets corrections as $G_{\mu \nu}^{(4)}+\Lambda g_{\mu \nu}^{(4)}+O(\lambda-1)+($ higher curvature corrections $)=8 \pi G_{N}\left(T_{\mu \nu}+\rho^{H L} n_{\mu} n_{\nu}\right)$.

The new terms in the left hand side result in extra contributions to the right hand side of (4.10) but they are again proportional to $n_{\nu}$ due to 3-dimensional spatial diffeomorphism invariance. Therefore, in general we have

$$
\partial_{\perp} \rho^{H L}+K \rho^{H L}=n^{\nu} \nabla^{\mu} T_{\mu \nu}+O(\lambda-1)+\text { (higher curvature corrections). }
$$

The right hand side is non-vanishing and the "dark matter" is inevitably "generated" if either the (real) matter sector or the gravity sector breaks a part of 4-dimensional diffeomorphism invariance. Thus, turning off the "dark matter" completely or, equivalently, imposing a local Hamiltonian constraint is not a consistent truncation in general.

In the early universe, the r.h.s. of (4.13) is non-vanishing. Quantum fluctuations of scalar graviton, tensor graviton and (real) matter fields act as source of "dark matter". 
Once a cosmological model is specified, it is possible to predict the typical amplitude of $\rho^{H L}$ generated in the early universe. (Such quantum fluctuations include modes with various wavelengths, including those much longer than the size of the present Hubble scale.) For this reason, the initial condition of "dark matter" is not arbitrary and this scenario has predictability.

On the other hand, at late time the r.h.s. of (4.13) should vanish if matter sector recovers 4-dimensional diffeomorphism in the IR and if $\lambda=1$ is a stable IR fixed point of the RG flow. In this case, (4.13) is reduced to the usual conservation law for "dark matter":

$$
\partial_{\perp} \rho^{H L}+K \rho^{H L}=0 .
$$

The flow of "dark matter" is tangent to the vector $n^{\mu}$ defined in (3.3) and thus is orthogonal to the constant time hypersurface. When a cusp is about to form, the spatial curvature of the constant time hypersurface increases. The system enters the non-relativistic regime and higher spatial curvature terms become important. Among them, terms with $z=3$ generate the strongest restoring force. Also, $\lambda$ may deviate from 1 by RG flow. As in some early universe models [4, [5, 8], we expect that the would-be cusp can bounce at short distance scales. Note that this is not because of deviation from geodesics 5 but because of repulsive gravity. In usual situation, congruence of geodesics would form caustics and thus cusps because gravity is attractive. On the other hand, for the flow of "dark matter" proposed in the present paper, higher curvature terms become important near the would-be cusps and provide negative effective energy and repulsive gravity. That is the reason why we expect bounce. Note that the bounce is provided by nonlinear terms and thus nonlinear analysis is needed. It is also important to include backreactions of the higher spatial curvature terms to the geometry since, as mentioned in the previous paragraph, the bounce is not due to deviation from geodesics but due to repulsive gravity at short distances. Without taking into account nonlinear terms and backreactions to the geometry, we would never be able to describe "dark matter" properly.

The initial value formulation of Hořava-Lifshitz gravity consists of dynamical equation, global Hamiltonian constraint, local momentum constraint and gauge conditions. In this language, the "dark matter" emerges only after solving the system of equations when we try to interpret a solution. Therefore, as far as scalar graviton, tensor graviton and matter fields are properly included as dynamical variables, we do not have to consider "dark matter" as an independent dynamical field. This structure

${ }^{5}$ In the case of ghost condensate [49, the derivative of the scalar field responsible for the condensate deviates from geodesics because of higher derivative terms [50]. On the other hand, in Hořava-Lifshitz gravity the vector $n^{\mu}$ always satisfies the geodesic equation (4.7). 
should persist even in quantum level. For this reason, we do not have to promote the "dark matter" from non-dynamical integration "constant" to a dynamical field. Nonetheless, when we interprete a solution after solving the dynamics of the system either classically or quantum mechanically, we see that difference from general relativity amounts to "dark matter".

\section{Summary and discussion}

In the non-relativistic, power-counting renormalizable theory of gravitation recently proposed by Hořava, the so called projectability condition must be respected as it stems from the fundamental symmetry of the theory, i.e. the foliation-preserving diffeomorphism. Essentially, the lapse function $N$ represents a gauge degree of freedom associated with space-independent time reparametrization. Thus, it is very natural to restrict $N$ to be independent of spatial coordinates. This is the projectability condition. Of course, a space-independent $N$ cannot be transformed to a space-dependent function by foliation-preserving diffeomorphism. The projectability condition then implies that the Hamiltonian constraint is not a local equation satisfied at each spatial point but an equation integrated over a whole space. This point was already made clear by Hořava in [1] (See footnote 4 of the present paper.).

Abandoning the projectability condition and imposing a local version of the Hamiltonian constraint would result in phenomenological obstacles [35] and theoretical inconsistencies [36]. Note that a strong self-coupling of the scalar graviton reported in [35] is not a problem if there is a phenomenon analogous to Vainshtein effect [44] since, unlike massive gravity [45] 6, Hořava-Lifshitz gravity is supposed to be UV complete. Other problems reported in [35, 36] disappear if the projectability condition is respected and if only the global Hamiltonian constraint is imposed. The Poisson brackets of constraints form a closed structure since there is only one Hamiltonian constraint and it commutes with itself [2]. The divergent coupling of the scalar graviton to matter source does not exist in the absence of local Hamiltonian constraint 7 . In conclusion, both theoretical consistencies and phenomenological viability require that the Hamiltonian constraint is not a local equation but an equation integrated over a whole space.

\footnotetext{
${ }^{6}$ In the case of massive gravity, Vainshtein effect [44] removes the vDVZ discontinuity [46, 47] but simultaneously makes quantum corrections uncontrollable [4]. For this reason, the massive gravity theory without a UV completion does not have predictability even at macroscopic scales.

${ }^{7}$ If the local Hamiltonian constraint were not used, then eq. (68) of [35] would not show a divergent coupling. This can be seen by moving the first term (written in term of $\dot{\sigma}$ ) in the r.h.s. to the l.h.s.
} 
The global Hamiltonian constraint is less restrictive than its local version, and allows a richer set of solutions than in general relativity. We have shown that a component which behaves like pressureless dust emerges as an "integration constant" of dynamical equations and momentum constraint equations. Consequently, classical solutions to the infrared limit of Hořava-Lifshitz gravity can mimic general relativity plus cold dark matter. The "dark matter" satisfies the (non-)conservation equation (4.13), whose source term is turned on if a part of 4-dimensional diffeomorphism invariance is broken by either the (real) matter sector or the gravity sector. In the IR, the source term vanishes and the standard conservation equation (4.14) holds. Also, the "modified Einstein equation" (4.12) leads to the Poisson equation (in a gauge with $N=N(t)$ ). Of course the "dark matter" can cluster.

\section{Note added}

After the present paper had appeared, the emergence of the "dark matter" was confirmed in several papers [51, 52, 53]. For example, eq. (22) of [53] is exactly equivalent to Einstein gravity plus cold dark matter. Obviously, it is not appropriate to interprete it as a scalar-tensor theory. (This is obvious if we notice that we usually do not try to interprete other cold dark matter models as scalar-tensor theories.) Nonetheless, ref. [53] did. Also, the analysis of ref. [53] is not capable of seeing discontinuity in the limit $\lambda \rightarrow 1$ since $\lambda$ is set to 1 from the beginning.

Ref. [52] has three statements about Hořava-Lifshitz gravity with the projectability condition: (i) "dark matter" forms caustics; (ii) "dark matter" is described by ghost condensate [49]; (iii) the scalar sector gets strongly coupled at the scale $\Lambda \sim \rho_{d}^{1 / 4}$ even with $\lambda \neq 1$. Actually, these three comments are not correct for the following reasons. (i) They did not take into account repulsive gravity due to nonlinear higher curvature terms (see the second-to-the-last paragraph in Sec. 4 of the present paper and also ref. [54]). (ii) Ghost condensate and Hořava-Lifshitz gravity have different symmetries. In particular, unlike ghost condensate, the invariance under (spaceindependent) time reparametrization forbids $h_{00}^{2}$ and thus $\dot{\pi}^{2}$. (iii) The strong coupling away from $\lambda=1$ found in [52] indicates breakdown of their description, i.e. the way dynamical degrees of freedom are identified, but does not imply inconsistency of the underlining UV theory. Note that the scalar graviton does not get strongly coupled away from $\lambda=1$ as is clear from e.g. eq. (54) of ref. [1]. See also the last paragraph in Sec. 4 of the present paper. When we take the limit $\lambda \rightarrow 1$, we have to take into account nonlinear interactions carefully to see if there is an analogue of the Vainshtein effect [44]. 
More recently, ref. [55] rediscovered ghost instability in the regime $1 / 3<\lambda<1$. This can be seen already in eq. (54) of [1] and just implies that we need to consider the region $\lambda>1$.

\section{Acknowledgements}

The author would like to thank Andrei Frolov, Keisuke Izumi, Kazuya Koyama, Miao Li, Hitoshi Murayama, Antonio Padilla, Oriol Pujolas, Keitaro Takahashi and Takahiro Tanaka for useful discussions. The work of the author was supported in part by MEXT through a Grant-in-Aid for Young Scientists (B) No. 17740134, by JSPS through a Grant-in-Aid for Creative Scientific Research No. 19GS0219, and by the Mitsubishi Foundation. This work was supported by World Premier International Research Center Initiative (WPI Initiative), MEXT, Japan.

\section{References}

[1] P. Horava, "Quantum Gravity at a Lifshitz Point," arXiv:0901.3775 [hep-th].

[2] P. Horava, "Membranes at Quantum Criticality," JHEP 0903, 020 (2009) arXiv:0812.4287 [hep-th]].

[3] T. Takahashi and J. Soda, "Chiral Primordial Gravitational Waves from a Lifshitz Point," arXiv:0904.0554 [hep-th].

[4] G. Calcagni, "Cosmology of the Lifshitz universe," arXiv:0904.0829 [hep-th].

[5] E. Kiritsis and G. Kofinas, "Horava-Lifshitz Cosmology," arXiv:0904.1334 [hepth].

[6] H. Lu, J. Mei and C. N. Pope, "Solutions to Horava Gravity," arXiv:0904.1595 [hep-th].

[7] S. Mukohyama, "Scale-invariant cosmological perturbations from Horava-Lifshitz gravity without inflation," arXiv:0904.2190 [hep-th].

[8] R. Brandenberger, "Matter Bounce in Horava-Lifshitz Cosmology," arXiv:0904.2835 [hep-th].

[9] H. Nikolic, "Horava-Lifshitz gravity, absolute time, and objective particles in curved space," arXiv:0904.3412 [hep-th]. 
[10] K. I. Izawa, "A Note on Quasi-Riemannian Gravity with Higher Derivatives," arXiv:0904.3593 [hep-th].

[11] H. Nastase, "On IR solutions in Horava gravity theories," arXiv:0904.3604 [hepth].

[12] R. G. Cai, L. M. Cao and N. Ohta, "Topological Black Holes in Horava-Lifshitz Gravity," arXiv:0904.3670 [hep-th].

[13] R. G. Cai, Y. Liu and Y. W. Sun, "On the $z=4$ Horava-Lifshitz Gravity," arXiv:0904.4104 [hep-th].

[14] Y. S. Piao, "Primordial Perturbation in Horava-Lifshitz Cosmology," arXiv:0904.4117 [hep-th].

[15] X. Gao, "Cosmological Perturbations and Non-Gaussianities in Hořava-Lifshitz Gravity," arXiv:0904.4187 [hep-th].

[16] E. O. Colgain and H. Yavartanoo, "Dyonic solution of Horava-Lifshitz Gravity," arXiv:0904.4357 [hep-th].

[17] T. Sotiriou, M. Visser and S. Weinfurtner, "Phenomenologically viable Lorentzviolating quantum gravity," arXiv:0904.4464 [hep-th].

[18] B. Chen and Q. G. Huang, "Field Theory at a Lifshitz Point," arXiv:0904.4565 [hep-th].

[19] S. Mukohyama, K. Nakayama, F. Takahashi and S. Yokoyama, "Phenomenological Aspects of Horava-Lifshitz Cosmology," arXiv:0905.0055 [hep-th].

[20] Y. S. Myung and Y. W. Kim, "Thermodynamics of Hořava-Lifshitz black holes," arXiv:0905.0179 [hep-th].

[21] R. G. Cai, B. Hu and H. B. Zhang, "Dynamical Scalar Degree of Freedom in Horava-Lifshitz Gravity," arXiv:0905.0255 [hep-th].

[22] D. Orlando and S. Reffert, "On the Renormalizability of Horava-Lifshitz-type Gravities," arXiv:0905.0301 [hep-th].

[23] C. Gao, "Modified gravity in Arnowitt-Deser-Misner formalism," arXiv:0905.0310 [astro-ph.CO].

[24] T. Nishioka, "Horava-Lifshitz Holography," arXiv:0905.0473 [hep-th]. 
[25] A. Kehagias and K. Sfetsos, "The black hole and FRW geometries of nonrelativistic gravity," arXiv:0905.0477 [hep-th].

[26] S. K. Rama, "Anisotropic Cosmology and (Super)Stiff Matter in Hořava's Gravity Theory," arXiv:0905.0700 [hep-th].

[27] R. G. Cai, L. M. Cao and N. Ohta, "Thermodynamics of Black Holes in HoravaLifshitz Gravity," arXiv:0905.0751 [hep-th].

[28] A. Ghodsi, "Toroidal solutions in Horava Gravity," arXiv:0905.0836 [hep-th].

[29] Y. S. Myung, "Thermodynamics of black holes in the deformed Hořava-Lifshitz gravity," arXiv:0905.0957 [hep-th].

[30] S. Chen and J. Jing, "Quasinormal modes of a black hole in the deformed HorravaLifshitz gravity," arXiv:0905.1409 [gr-qc].

[31] J. Kluson, "Stable and Unstable D-Branes at Criticality," arXiv:0905.1483 [hepth].

[32] R. A. Konoplya, "Towards constraining of the Horava-Lifshitz gravities," arXiv:0905.1523 [hep-th].

[33] S. Chen and J. Jing, "Strong field gravitational lensing in the deformed HorravaLifshitz black hole," arXiv:0905.2055 [gr-qc].

[34] B. Chen, S. Pi and J. Z. Tang, "Scale Invariant Power Spectrum in HořavaLifshitz Cosmology without Matter," arXiv:0905.2300 [hep-th].

[35] C. Charmousis, G. Niz, A. Padilla and P. M. Saffin, "Strong coupling in Horava gravity," arXiv:0905.2579 [hep-th].

[36] M. Li and Y. Pang, "A Trouble with Hořava-Lifshitz Gravity," arXiv:0905.2751 [hep-th].

[37] T. P. Sotiriou, M. Visser and S. Weinfurtner, "Quantum gravity without Lorentz invariance," arXiv:0905.2798 [hep-th].

[38] Y. W. Kim, H. W. Lee and Y. S. Myung, "Nonpropagation of scalar in the deformed Hořava-Lifshitz gravity," arXiv:0905.3423 [hep-th].

[39] E. N. Saridakis, "Horava-Lifshitz Dark Energy," arXiv:0905.3532 [hep-th]. 
[40] G. Calcagni, "Detailed balance in Horava-Lifshitz gravity," arXiv:0905.3740 [hepth].

[41] S. R. Coleman and S. L. Glashow, "High-Energy Tests of Lorentz Invariance," Phys. Rev. D 59, 116008 (1999) arXiv:hep-ph/9812418.

[42] T. Jacobson, S. Liberati and D. Mattingly, "TeV astrophysics constraints on Planck scale Lorentz violation," Phys. Rev. D 66, 081302 (2002) arXiv:hep-ph/0112207.

[43] G. D. Moore and A. E. Nelson, "Lower bound on the propagation speed of gravity from gravitational Cherenkov radiation," JHEP 0109, 023 (2001) arXiv:hep-ph/0106220.

[44] A. I. Vainshtein, "To the problem of nonvanishing gravitation mass," Phys. Lett. B 39, 393 (1972).

[45] M. Fierz and W. Pauli, "On relativistic wave equations for particles of arbitrary spin in an electromagnetic field," Proc. Roy. Soc. Lond. A 173, 211 (1939).

[46] H. van Dam and M. J. G. Veltman, "Massive And Massless Yang-Mills And Gravitational Fields," Nucl. Phys. B 22, 397 (1970).

[47] V. I. Zakharov, "Linearized gravitation theory and the graviton mass," JETP Lett. 12, 312 (1970) [Pisma Zh. Eksp. Teor. Fiz. 12, 447 (1970)].

[48] N. Arkani-Hamed, H. Georgi and M. D. Schwartz, "Effective field theory for massive gravitons and gravity in theory space," Annals Phys. 305, 96 (2003) arXiv:hep-th/0210184.

[49] N. Arkani-Hamed, H. C. Cheng, M. A. Luty and S. Mukohyama, "Ghost condensation and a consistent infrared modification of gravity," JHEP 0405, 074 (2004) arXiv:hep-th/0312099.

[50] N. Arkani-Hamed, H. C. Cheng, M. A. Luty, S. Mukohyama and T. Wiseman, "Dynamics of Gravity in a Higgs Phase," JHEP 0701, 036 (2007) arXiv:hep-ph/0507120.

[51] A. A. Kocharyan, "Is nonrelativistic gravity possible?," arXiv:0905.4204 [hep-th].

[52] D. Blas, O. Pujolas and S. Sibiryakov, "On the Extra Mode and Inconsistency of Horava Gravity," arXiv:0906.3046 [hep-th]. 
[53] A. Kobakhidze, "On the infrared limit of Horava's gravity with the global Hamiltonian constraint," arXiv:0906.5401 [hep-th].

[54] S. Mukohyama, "Caustic avoidance in Horava-Lifshitz gravity," arXiv:0906.5069 [hep-th].

[55] C. Bogdanos and E. N. Saridakis, "Perturbative instabilities in Horava gravity," arXiv:0907.1636 [hep-th]. 